hep-th/9508073

SLAC-PUB-95-6986

1995

\title{
Grand Unification with Three Generations in Free Fermionic String Models
}

\author{
D. Finnell ${ }^{1}$ \\ Stanford Linear Accelerator Center \\ Stanford University, Stanford, California 94309, USA
}

\begin{abstract}
We examine the problem of constructing three generation free fermionic string models with grand unified gauge groups. We attempt the construction of $G \times G$ models, where $G$ is a grand unified group realized at level 1. This structure allows those Higgs representations to appear which are necessary to break the symmetry down to the standard model gauge group. For $G=S O(10)$, we find only models with an even number of generations. However, for $G=S U(5)$ we find a number of 3 generation models.
\end{abstract}

${ }^{1}$ Address after Sept. 1, 1995: Deptartment of Physics and Astronomy, Rutgers University, Piscataway NJ 08855-0849. 


\section{Introduction}

The apparent unification of the gauge couplings of the MSSM [1] is a successful prediction of supersymmetric grand unified theories. In string theory, the unification of coupling constants is also predicted [2], although at a scale about one order of magnitude higher than the $3 \cdot 10^{16} \mathrm{GeV}$ indicated by experiment [3]. This discrepancy may be the result of threshold corrections or charged matter at an intermediate scale modifying the naive prediction (for a recent review see [4]), or a nonstandard normalization of the $U(1)$ hypercharge [5]. It is also possible that the string theory leads to a grand unified group which is spontaneously broken below the string scale. This has motivated a search for realistic string models featuring grand unified (GUT) groups.

It has been known for some time that the incorporation of grand unification within string theory poses challenges. The most basic representations of gauge symmetries on the string world sheet, the $k=1$ representations, do not allow for scalars in the adjoint representation, necessary for the breaking of GUT symmetry, at the same time as chiral fermions [6, [7, 8]. This problem can be overcome by the construction of string models with gauge groups realized at $k \geq 2$. Although such constructions are technically complicated, models have been constructed both in the orbifold and free-fermion formulations which feature the basic ingredients of a realistic GUT group $(S O(10)$ or $S U(5))$ adjoint Higgs scalars and chiral fermions 8, 9, 10].

One feature which has proven difficult to obtain in these models is the correct three-fold replication of chiral fermion families. To date, a variety of non-unified or semi-unified level 1 three generation string models have been constructed in the literature $111,12,13$, 14, 15, 16]. Methods for constructing such models in the free-fermion or orbifold frameworks are well established. However, despite effort by a number of groups [9, 10, 18, 19], there exist no examples in the literature of consistent string GUT models with three generations.

In this paper, we will focus on the specific problem of constructing a string model which combines grand unification with three generations of chiral fermions. We will approach the problem from a practical point of view, attempting explicit constructions using the free-fermionic formulation for string compactifications. Rather than constructing $k=2$ models, we will follow the suggestion of [17, 20, 19] and construct models which con- 
tain a direct product of gauge factors $G_{i} \geq S U(5)$, each realized at level 1 , with massless scalars transforming as vectors simultaneously under two groups. Vacuum expectation values (VEV's) for these fields can then break the gauge group down to the standard model embedded in a diagonal subgroup. For example, we will attempt to build $S O(10) \times S O(10)$ models with a $(\mathbf{1 0}, \mathbf{1 0})$ representation, or $S U(5) \times S U(5)$ with $(\mathbf{5}, \overline{\mathbf{5}})+(\overline{\mathbf{5}}, \mathbf{5})$. A VEV along the D-flat direction

$$
\left\langle H_{i \bar{j}}\right\rangle=\left\langle\bar{H}_{\bar{i} j}\right\rangle=\operatorname{diag}(X, X, X, Y, Y) .
$$

with $X \simeq Y \simeq M_{\mathrm{GUT}}, Y \neq X$, would break $S U(5) \times S U(5)$ down to the diagonal subgroup $(S U(3) \times S U(2) \times U(1))_{D}$, and the three standard model gauge couplings would be unified at $X$, even if the couplings of the two $S U(5)$ factors were not equal. This mimics a conventional GUT group, with the mixed representations $H, \bar{H}$ playing the role of the adjoint Higgs. For these reasons we will refer to these models as "unified" models even though the GUT group is not simple. The fact that such models can be built with level 1 representations allows us to avoid much of the technical baggage associated with higher level models and concentrate on obtaining three generations. Sting models featuring this construction have been investigated extensively by Maslikov et. al. [19.

We will organize our discussion as follows. In Sections 2 and 3, we will review the technique employed in constructing level 1 three generation free fermion models, constructing $S O(10)$ and $S U(5)$ models without Higgs

scalars as a demonstration. In Section 4, we will apply this technique to models with the enlarged gauge sector $S O(10) \times S O(10)$ with $(\mathbf{1 0}, \mathbf{1 0})$ Higgs representations, and study the complications which arise. Finally, in Section 5 , we will identify a construction which allows us to obtain a three generation spectrum in $S U(5) \times S U(5)$ models, and we will present a number of explicit models.

\section{Free Fermion Conventions}

In free fermion models, the string degrees of freedom needed to cancel the conformal anomaly consist of 20 real right moving (10 complex) and 44 real (22 complex) left moving free fermions. The rules for the construction of consistent free fermion string models were worked out in [21, 22]. We will follow 
the conventions of [21], where the boundary conditions imposed upon free fermions under parallel transport about noncontractable loops are expressed in terms of vectors $V$ such that $\Psi^{l} \rightarrow-e^{-2 \pi i V^{l}} \Psi^{l}$, where $-\frac{1}{2} \leq V_{i}^{l}<\frac{1}{2}$. The boundary conditions are generated by a set of basis vectors $V_{i}$. Let $m_{i}$ be the smallest integer for which $m_{i} V_{i}=0(\bmod 1)$. Then the basis vectors must satisfy

$$
\begin{aligned}
m_{i} V_{i} \cdot V_{j}=m_{j} V_{i} \cdot V_{j} & =0 \quad(\bmod 1) \\
2 m_{i} V_{i} \cdot V_{i} & =0 \quad(\bmod 1),
\end{aligned}
$$

where dot products are defined with Lorentzian signature

$$
V_{i} \cdot V_{j}=-\sum_{\text {right }} \delta_{l} V_{i}^{l} V_{j}^{l}+\sum_{\text {left }} \delta_{l} V_{i}^{l} V_{j}^{l}
$$

( $\delta_{l}=1 / 2$ for a real fermion, 1 for a complex one). Phases appearing in the partition function are defined in terms of an auxiliary matrix $k_{i j}$, satisfying

$$
\begin{aligned}
k_{i j}+k_{j i} & =V_{i} \cdot V_{j}(\bmod 1) \\
m_{j} k_{i j} & =0(\bmod 1) \\
k_{i i}+k_{i 0}+s_{i}-\frac{1}{2} V_{i} \cdot V_{i} & =0(\bmod 1)
\end{aligned}
$$

Note that $s_{i}$ represents the boundary condition for the spacetime free fermion. To specify a string model, one must specify both the set of basis vectors and the $k$-matrix. We will follow the convention of stating the nonvanishing lower half-diagonal $k$-matrix elements, allowing the full $k$-matrix to be reconstructed using the above equations.

Every set of boundary conditions generated by linear combinations of the basis vectors defines a different sector of the Hilbert space of string states. States to be identified with physical particles must satisfy $m^{2}=H^{L}=H^{R}$, where $H^{L}, H^{R}$ are the left and right moving worldsheet Hamiltonians. The vacuum energy of $H^{L}$ is

$$
E_{0}^{L, V}=-\frac{1}{12}+\sum_{l, \text { left }} \frac{1}{2}\left(\left(V^{l}\right)^{2}-\frac{1}{12}\right) \delta_{l} .
$$

and a similar equation holds for $E_{R}$. Physical states must also satisfy a generalized GSO projection for each basis vector:

$$
V_{i} \cdot N_{\alpha V}=\sum_{j} k_{i j} \alpha_{j}+s_{i}-V_{i} \cdot \overline{\alpha V} \quad(\bmod 1)
$$


where $N_{\alpha V}$ is the vector of fermion excitations in the sector $V=\sum_{i} \alpha_{i} V_{i}$.

Following standard notation, taking a " 1 " to represent $V=-1 / 2$, we take

$$
V_{0}=\left(1^{20} \mid 1^{44}\right)
$$

as our first basis vector. We will choose as our second basis vector the conventional choice for the gravitino sector

$$
V_{1}=\left(1^{2}(100)^{6} \mid 0^{44}\right) .
$$

We choose the standard form of the worldsheet supercurrent

$$
T_{F}=\bar{\psi}^{\mu} \partial X_{\mu}+i \sum_{I} \bar{\chi}_{I} \bar{y}_{I} \bar{\omega}_{I}
$$

which must also be preserved by boundary conditions. We will use only periodic and antiperiodic boundary conditions for the supersymmetric side of the string, for which the "triplet constraint" [21]

$$
V_{j}\left(\bar{\chi}_{i}\right)+V_{j}\left(\bar{y}_{i}\right)+V_{j}\left(\bar{\omega}_{i}\right)=s_{j}(\bmod 1),
$$

is sufficient to guarantee superconformal invariance.

\section{The standard mechanism for three genera- tions}

The three generation free fermion models existing in the literature are based on a set of basis vectors known as the "NAHE" set 12, 14]:

$$
\begin{aligned}
& V_{2}=\left(1_{c}(100)^{2}(010)^{2}(010)^{2} \mid(00)^{2}(10)^{2}(10)^{2} 1^{10}(100)_{c} 0^{16}\right) \\
& V_{3}=\left(1_{c}(010)^{2}(100)^{2}(001)^{2} \mid(10)^{2}(00)^{2}(01)^{2} 1^{10}(010)_{c} 0^{16}\right) \\
& V_{4}=\left(1_{c}(001)^{2}(001)^{2}(100)^{2} \mid(01)^{2}(01)^{2}(00)^{2} 1^{10}(001)_{c} 0^{16}\right)
\end{aligned}
$$

where we have used the notation

$$
V_{i}=\left(S_{i}\left(\bar{\chi}_{1} \bar{y}_{1} \bar{\omega}_{1}\right) \ldots \mid\left(y_{1} \omega_{1}\right) \ldots \Psi_{a}^{10}\left(\eta_{1} \eta_{2} \eta_{3}\right)_{c} \Psi_{b}^{16}\right) .
$$

The subscript " $c$ " denotes boundary conditions for complex, as opposed to real, fermions. The group of ten fermions with identical boundary conditions, 
$\Psi_{a}^{10}$, leads to an $S O(10)$ gauge group, while $\Psi_{b}^{16}$ generate an $S O(16)$ group (which may be enhanced to $E_{8}$ by gauge bosons from twisted sectors). Also note, at this stage, each $\eta_{i}$ will pair up with some $y_{i}$ 's and $\omega_{i}$ 's to form an $S O(6)^{3}$ horizontal gauge group. Thus the total gauge group is $S O(10) \times$ $E_{8} \times S O(6)^{3}$. This set of basis vectors has been used as the starting point for standard-like and "flipped" $S U(5)$ models; the origin of three generations in these models is studied in [23, 24]. It will be instructive to ignore for the moment the complications which follow from the need for adjoint Higgs scalars and see what is involved in building a three generation $S O(10)$ (or $S U(5))$ model based on the above basis vectors.

The above three basis vectors represent the three distinct classes of righthand-side boundary conditions one can have, which correspond to the three twists of the $Z_{2} \times Z_{2}$ orbifold [24] (+- ), (-+-), and (--+) respectively, acting on three 2 -tori. This set of twists breaks the $N=4$ supersymmetry to $N=1$. In the standard approach, these three distinct twists are to be the origin of three generations, one generation arising from each. Each of the above sectors, $V_{2,3,4}$, has been chosen to give massless chiral fermions in the 16 representation of $S O(10)$. Take sector $V_{2}$ for example. The vacuum energy is $\left(E_{R}, E_{L}\right)=(0,0)$, so unexcited states from this sector are massless. The fermion zero modes of $\Psi_{a}^{10}$ realize spinor representations of $S O(10)$ (16's and $\overline{\mathbf{1 6}}$ 's). However, there are additional zero modes, the zero modes of $S_{i}, \bar{\chi}_{1,2}$, $\bar{y}_{3-6}, y_{3-6}$, and $\eta_{1}$. Prior to the GSO projections, the original Clifford vacuum has dimension $2^{11}=2^{6} \cdot \mathbf{1 6}+2^{6} \cdot \overline{\mathbf{1 6}}$ for each spacetime chirality. We can group the anticommuting fermion zero modes into sets of commuting operators, $\bar{\Gamma}^{0}=S^{\dagger} S, \Gamma^{10}=i \prod_{a} \Psi_{a}^{10}, i \bar{\chi}_{1} \bar{\chi}_{2}, i \bar{y}_{I} y_{I}$, and $\eta_{1}^{\dagger} \eta_{1}$, whose eigenvalues will label the degenerate states. Modulo 1, the eigenvalues of these are related to fermion excitation numbers appearing in Eq. (9) by [21]

$$
\frac{1}{2}\left(1-\Gamma_{i}\right)=N_{i} \bmod 1 .
$$

The eigenvalue of $\Gamma^{10}$ determines the $S O(10)$ chirality (16 vs. $\overline{\mathbf{1 6}}$ ), while that of $\bar{\Gamma}^{0}$ determines the spacetime chirality. The GSO projection due to $V_{3}$ (or $V_{4}$ ) (Eq. (9)) correlates $S O(10)$ chirality with spacetime chirality for massless states; only left-handed 16's and right handed $\overline{\mathbf{1 6}}$ 's survive from this sector. The value of $i \bar{\chi}_{1} \bar{\chi}_{2}$ is also tied to spacetime chirality. We are left with the five internal fermion operators $i \bar{y}_{i} y_{i}, i=3 \ldots 6$, and $\eta_{1}^{\dagger} \eta_{1}$, satisfying the constraint $\eta^{\dagger} \eta \prod_{i}\left(i \bar{y}_{i} y_{i}\right)=+1$. This leaves an overall degeneracy of $2^{5-1}=16$ 
for every chiral 16. They can be shown to transform as two copies of $4+\overline{4}$ under the $S O(6)$ horizontal groups.

At this stage, the model has a total of 48 generations. To reduce this number, one must add additional basis vectors, such that only one replication of 16 survives GSO projections in each of the above sectors. In choosing additional basis vectors, it is simplest to preserve the pairing between left and right moving fermions, $V\left(\bar{y}^{I}\right)=V\left(y^{I}\right), V\left(\bar{\omega}^{I}\right)=V\left(\omega^{I}\right)$. We could alternately choose boundary conditions which broke this pairing, instead pairing on the left and right sides separately giving models with complex fermions]. Because of the Lorentzian signature of the dot product, Eq. 目, the left-right paired basis vectors automatically satisfy Eqs. 3. We may choose, for example:

$$
\begin{aligned}
& V_{5}=\left(0_{c} 011000000000000011 \mid 1100000000110^{10} 0_{c}^{3} 0^{16}\right) \\
& V_{6}=\left(0_{c} 000011011000000000 \mid 0011110000000^{10} 0_{c}^{3} 0^{16}\right) \\
& V_{7}=\left(0_{c} 000000000011011000 \mid 0000001111000^{10} 0_{c}^{3} 0^{16}\right) \text {. }
\end{aligned}
$$

This set of twists in the internal fermions breaks the horizontal symmetry $S O(6)^{3} \rightarrow U(1)^{6}$; the six remaining $U(1)$ currents are $\eta_{i}^{\dagger} \eta_{i}$, and three currents associated with the internal fermions, $J_{1}=i y_{4} y_{5}, J_{2}=i y_{1} \omega_{6}, J_{3}=i \omega_{2} \omega_{3}$. There is now only a two-fold degeneracy, corresponding to the two different eigenvalues of the current $i y_{4} y_{5}= \pm 1 . V_{3}$ and $V_{4}$ are similar, each having a $\mathbf{1 6}_{ \pm}$under their corresponding $U(1)$ 's. This model now has 6 generations. Note that, with the addition of these basis vectors, new sectors containing massless nonchiral $\mathbf{1 6}+\overline{\mathbf{1 6}}$ pairs have appeared, for example $V_{2}+V_{7}, V_{3}+V_{5}$ and $V_{4}+V_{6}$.

To make the final reduction to three generations, we can attempt to add another left-right symmetric vector which breaks down the internal $U(1)$ 's labeling the degenerate $\mathbf{1 6}$ 's. Such a vector is, for example:

$$
V_{8}=\left(0_{c} 011000011000011000 \mid 1100110011000^{10} 0_{c}^{3} 0^{16}\right) .
$$

This vector indeed leaves only one $\mathbf{1 6}$ from each of the three sectors $V_{2}, V_{3}, V_{4}$. However, we find the unpleasant feature that new, unwanted chiral 16's have popped up in other sectors. In this example, with the minimal $k$-matrix $\left(k_{21}=k_{31}=k_{41}=1 / 2\right)$, sectors $V_{2}+V_{5}+V_{7}+V_{8}, V_{3}+V_{5}+V_{6}+V_{8}$ and

\footnotetext{
${ }^{1} \mathrm{~A}$ third choice [8, 9], taking fermions with unpairably real boundary conditions, will not be used here.
} 
$V_{4}+V_{6}+V_{7}+V_{8}$ yield $\overline{\mathbf{1 6}}$ 's, resulting in 0 net chiral generations. Changing the $k$-matrix to $k_{72}=k_{74}=k_{83}=1 / 2$ we find 16 's in $V_{2}+V_{5}+V_{8}, V_{3}+V_{6}+V_{8}$ and a $\overline{\mathbf{1 6}}$ in $V_{4}+V_{7}+V_{8}$, for 4 net generations. For all allowed choices of $k_{i j}$ the number of generations is even.

The appearance of unwanted states when new projections are added is the complication that makes obtaining three generations so challenging. One strategy for curing this problem is to include, in the basis vectors added after the NAHE set, additional twists for the "hidden sector" fermions. Since Ramond boundary conditions raise the vacuum energy of a sector (Eq. 8), this will have the effect of raising the mass of states from sectors involving this twist. Such a twist will not, however, be left-right symmetric, and the constraints of Eqs. 3 will be more restrictive. For real fermions with $0,1 / 2$ boundary conditions, the number of (real) unpaired 1/2's must be equal to $0 \bmod 8$, and the number of (real) 1/2's overlapping between two different vectors must be 0 mod 4 . A sample solution is

$$
\begin{aligned}
& V_{7}=\left(\begin{array}{ll|l}
0_{c} 000000000011011000 & 0000001111000^{10} 0_{c}^{3} 0^{4} 1^{4} 1^{4} 0^{4}
\end{array}\right) \\
& V_{8}=\left(\begin{array}{lll}
0_{c} 011000011000011000 & 1100110011000^{10} 0_{c}^{3} 1^{4} 1^{4} 0^{4} 0^{4}
\end{array}\right) .
\end{aligned}
$$

These vectors remove the unwanted massless states and leave precisely three 16's. Note the price we had to pay was breaking apart our "hidden sector", which was broken down to $S O(4)^{4}$ (although it can be enhanced by twisted sector gauge bosons).

An alternate mechanism for breaking the degeneracy is, instead of breaking the horizontal $U(1)$ currents, to break their pairing with right-moving counterparts. We may add a twist which is embedded in the internal $U(1)_{i}$ 's, which label the two 16's in a given sector, but not their right-moving counterparts. As this is left-right asymmetric, the constraints in Eqs. 3 will be more difficult to satisfy; in fact, we cannot satisfy them with only $0,1 / 2$ boundary conditions. To find solutions, we must include $1 / 4$ twists, which loosen the constraints somewhat $\left(4 V_{i} \cdot V_{j}=0 \bmod 1\right.$ instead of $\left.2 V_{i} \cdot V_{j}=0 \bmod 1\right)$. However, the $V \cdot V$ constraint Eq. 通, will restrict the number of $1 / 4$ boundary conditions which can appear. We must have

$$
4 V \cdot V=N_{\frac{1}{2}}+\frac{1}{4} \cdot N_{\frac{1}{4}}=0 \quad(\bmod 2),
$$

where $N_{\frac{1}{2}}, N_{\frac{1}{4}}$ are the number of complex fermions with $1 / 2$ and $1 / 4$ boundary conditions respectively. If we wish the vector to include 3 asymmetric 
$1 / 2$ 's, then the number of $1 / 4$ 's must be an odd multiple of 4 . Thus, letting "+" represent $V=1 / 4$, we add the vector

$$
V_{7}=\left(0(000)^{6} \mid 100101101001+{ }_{c}^{5}+{ }_{c}^{3}+{ }_{c}^{4} 0_{c}^{4}\right)
$$

to $V_{0}-V_{6}$. The $1 / 4$ 's in this vector break the $S O(10)$ gauge group to $S U(5)$. In the process, the $\mathbf{1 6}$ 's are broken into their components $\mathbf{1}+\mathbf{1 0}+\overline{\mathbf{5}}$ under $S U(5)$. The equation determining which states survive projection is:

$$
\frac{1}{2} N_{J}+\frac{1}{4} N_{5}=\sum_{i} k_{7 i} \alpha_{i} \quad(\bmod 1)
$$

where $N_{J}$ is the excitation number for the internal $U(1)$ labeling the degenerate 16's, and $N_{5}$ is the excitation number in the $S U(5)$ sector, equal to 0 for the $\mathbf{1}, 2$ for the $\mathbf{1 0}$ and 4 for the $\overline{\mathbf{5}}$. Thus, assuming $\sum_{i} k_{7 i}=0$, from $\mathbf{1 6}_{ \pm}$, a $\mathbf{1}+\overline{\mathbf{5}}$ survives from $16_{+}$and a $\mathbf{1 0}$ from $16_{-}$, leaving one complete $S U(5)$ multiplet from each of three sectors. Note that to preserve modular invariance, we were forced to break the hidden sector gauge group also to $U(4) \times S O(8)$. This is the mechanism used to build three generation "flipped" $S U(5)$ models [12]. Note that, in both this approach and the previous one, the "hidden" sector fermions played an important role in allowing us to make the final reduction to three generations.

\section{$4 \quad S O(10) \times S O(10)$ Models}

To attempt a generalization of the above approaches to a unified model with adjoint Higgs representations, we must do two things: enlarge the gauge group to $S O(10) \times S O(10)$, and insure that mixed Higgs representations such as $(\mathbf{1 0}, \mathbf{1 0})$ actually exist. The first is easy to accomplish; we just take a second $S O(10)$ from the $S O(16)$ hidden sector of the above models. However, the second feature requires a substantial modification. This Higgs

representation transforms as a vector under two gauge groups simultaneously; it must be created by exciting two fermions, one carrying the vector index under each group, entailing a large excitation energy. If we limit ourselves to $0,1 / 2$ boundary conditions, both fermions must have NS boundary conditions, and the energy associated with the two fermion excitations will be +1 . For the state to be massless, it must therefore arise from a sector with 
ground state energy $E_{L}=-1$. The natural candidate for this sector is the Neveu-Schwarz sector, $V=0$, with $\left(E_{R}, E_{L}\right)=(-1 / 2,-1)$. We will attempt to arrange our basis vector such that there is a surviving state of the form

$$
\bar{\chi}_{-1 / 2}^{I}|0\rangle_{R} \Psi_{-1 / 2}^{A} \Psi_{-1 / 2}^{B}|0\rangle_{L}
$$

which transforms as a $(\mathbf{1 0}, \mathbf{1 0})$ under $S O(10)_{A} \times S O(10)_{B}$.

We immediately run into a problem, though, with the set of basis vectors used in the previous section, the NAHE set. This set of vectors automatically projects out any states in the NS sector which would transform under both the original $S O(10)$ and a group coming from the hidden sector. Explicitly, the projections must include the sum

$$
V_{0}+V_{2}+V_{3}+V_{4}=\left(0_{c} 0^{18} \mid 0^{12} 0^{10} 0_{c}^{3} 1^{16}\right) .
$$

This enforces a projection that requires an even number of fermionic excitations in the $S O(16)$ sector, thus eliminating the mixed states. Clearly we must modify this set of vectors.

The fact that mixed representations are projected out of the untwisted sector is a manifestation of the fact that models based on the NAHE set are compactifications of the $E_{8} \times E_{8}$ string. Progress can be made by finding a set of vectors which correspond instead to a compactification of the $S O(32)$ stringfl. This is accomplished with the set:

$$
\begin{aligned}
V_{2}=\left(1_{c}(100)^{2}(010)^{2}(010)^{2}\right. & \left.\mid(00)^{2}(10)^{2}(10)^{2} 1^{10} 0^{10} 0^{10} 1_{c}\right) \\
V_{3}=\left(1_{c}(010)^{2}(100)^{2}(001)^{2}\right. & \left.\mid(10)^{2}(00)^{2}(01)^{2} 0^{10} 1^{10} 0^{10} 1_{c}\right) \\
V_{P}=\left(0_{c}(000)^{2}(000)^{2}(000)^{2}\right. & \left.\mid(00)^{2}(00)^{2}(00)^{2} 1^{10} 1^{10} 1^{10} 1_{c}\right) .
\end{aligned}
$$

$V_{0}, V_{1}$ and $V_{P}$ produce a toroidal compactification of the $S O(32)$ string (with background fields). The vectors $V_{2}, V_{3}$ provide the $Z_{2} \times Z_{2}$ orbifold twists that reduce the $N=4$ supersymmetry to $N=1$, as was the case for the NAHE set. However, they differ now in that each twist is embedded in a different $S O(10)$ factor, in the process breaking $S O(32) \rightarrow S O(10)^{3} \times U(1)$. The vector $V_{P}$ is just the standard GSO projection of the ten dimensional $S O(32)$ string.

\footnotetext{
${ }^{2}$ The advantage of using the $S O(32)$ string has also been observed in 10$]$.
} 
An examination of the projections in the NS sector now reveal that the states

$$
\begin{aligned}
& \bar{\chi}_{-1 / 2}^{1,2}|0\rangle_{R} \Psi_{-1 / 2}^{10 A} \Psi_{-1 / 2}^{10 B}|0\rangle_{L} \\
& \bar{\chi}_{-1 / 2}^{3,4}|0\rangle_{R} \Psi_{-1 / 2}^{10 A} \Psi_{-1 / 2}^{10 C}|0\rangle_{L}
\end{aligned}
$$

and

$$
\bar{\chi}_{-1 / 2}^{5,6}|0\rangle_{R} \Psi_{-1 / 2}^{10 B} \Psi_{-1 / 2}^{10 C}|0\rangle_{L}
$$

survive, providing the full set $(\mathbf{1 0}, \mathbf{1 0}, 1)+(\mathbf{1 0}, 1, \mathbf{1 0})+(1, \mathbf{1 0}, \mathbf{1 0})$ necessary for breaking $S O(10)^{3} \rightarrow S O(10)_{D}$.

The sectors $V_{2}, V_{3}$, and $V_{0}+V_{2}+V_{3}$ provide $(\mathbf{1 6}, 1,1),(1, \mathbf{1 6}, 1),(1,1, \mathbf{1 6})$ representations respectively, becoming chiral generations under $S O(10)_{D}$. Each is replicated 8 times per sector. As in the previous section, we may reduce this degeneracy by adding left-right paired vectors which break the horizontal symmetries. Adding

$$
\begin{aligned}
& V_{4}=\left(\begin{array}{ll|l}
0_{c} 011000000000000011 & 1100000000110^{10} 0_{c}^{3} 0^{16}
\end{array}\right) \\
& V_{5}=\left(\begin{array}{llll}
0_{c} 000011011000000000 & 00111100000000^{10} 0_{c}^{3} 0^{16}
\end{array}\right)
\end{aligned}
$$

breaks down the horizontal symmetry to $U(1)^{3}$, and leaves a 2-fold degeneracy in each sector associated with the generational $U(1)$ charge $i y_{4} y_{5}$ for $V_{2}$, $i y_{1} \omega_{6}$ and $i \omega_{2} \omega_{3}$ for $V_{3}$ and $V_{2}+V_{3}+V_{0}$ respectively. This again leaves a total of six chiral generations. Adding further projections, to reduce this number, again produces unwanted states in new sectors. In the previous section, we saw that this could be circumvented by making proper use of the hidden sector. However, in these models, with the extended gauge sector, there is essentially no hidden sector left, and we cannot apply the tricks used in the previous section. All examples we have constructed have an even number of generations.

All our attempts to use the standard $Z_{2} \times Z_{2}$ mechanism for generating three generation models fail, and we must look for an alternate method. We will take the approach of only using two of the three $Z_{2} \times Z_{2}$ twisted sectors for chiral generations, and not attempting to treat the different sectors symmetrically (i.e., have two generations associated with one twist, one with the other). We start with the basic set defined above, producing $S O(10)^{3}$, with 8 16's per sector. We will add additional vectors which preserve the first two $S O(10)$ 's, and preserve the $(\mathbf{1 0}, \mathbf{1 0})$ representations in the untwisted 
sector, but we will allow ourselves the freedom to break apart the third $S O(10)$.

The number of massless 16 's coming from a given sector is $2^{7-n_{i}}$ where $n_{i}$ is the number of independent projections imposed on the zero modes by the generalized GSO projections (assuming 0,1/2 boundary conditions). Since this is a power of two, if we are going to have an odd number of 16's (three), there must be at least one sector for which $n_{i}=7$. This requires that at least three additional vectors be added to the above set.

We will attempt to add additional vectors such that sector $V_{3}$ has $n=7$ and $V_{2}$ has $n=6$. We add, to $V_{2}, V_{3}, V_{P}$, the vectors

$$
\begin{aligned}
& V_{4}=\left(\begin{array}{ll|l}
0_{c} 011000000000000011 & 1100000000110^{10} & 0^{10}\left(0^{4} 0^{2}\right)_{c}
\end{array}\right) \\
& V_{5}=\left(\begin{array}{llllll}
0_{c} 000011011000000000 & 001111000000 & 0^{10} & 0^{10}\left(1^{4} 0^{2}\right)_{c}
\end{array}\right) \\
& V_{6}=\left(\begin{array}{lllll}
0_{c} 011000011000000000 & 1100110000000^{10} & 0^{10}\left(0^{4} 0^{2}\right)_{c}
\end{array}\right) .
\end{aligned}
$$

With this set of basis vectors, the gauge group has been broken to $S O(10)^{2} \times$ $S O(8) \times U(1)^{3}$. Unfortunately, the available "hidden sector" has not provided enough freedom to inhibit additional chiral sectors from appearing. We find a total of four sectors for which $n_{i}=7$; writing them as $V=\sum_{i} \alpha_{i} V_{i}$ these sectors are

$$
\begin{aligned}
& \vec{\alpha}^{1}=(0,0,0,1,0,0,0,0) \\
& \vec{\alpha}^{2}=(0,0,0,1,0,1,1,1) \\
& \vec{\alpha}^{3}=(0,0,0,1,1,0,0,0) \\
& \vec{\alpha}^{4}=(0,0,0,1,1,1,1,1)
\end{aligned}
$$

where

$$
\vec{\alpha}=\left(\alpha_{0}, \alpha_{1}, \alpha_{2}, \alpha_{3}, \alpha_{4}, \alpha_{5}, \alpha_{6}, \alpha_{P}\right) .
$$

In addition, there are four sectors for which $n_{i}=6$,

$$
\begin{aligned}
\vec{\alpha}^{5} & =(0,0,1,0,0,0,0,0) \\
\vec{\alpha}^{6} & =(0,0,1,0,1,0,1,0) \\
\vec{\alpha}^{7} & =(1,1,1,0,0,1,1,0) \\
\vec{\alpha}^{8} & =(1,1,1,0,1,1,0,0) .
\end{aligned}
$$

We refer to these as being degenerate sectors, because they produce multiple 16's. In these sectors, some of the projections on the massless states in 
these sectors are redundant. Then, if the $k$-matrix is chosen such that the redundant projections are incompatible, all massless states from the sector can be eliminated. However, eliminating one of these sectors only changes the net number of chiral 16's, $N(\mathbf{1 6})-N(\overline{\mathbf{1 6}})$, by an even number (two). For the sectors $\vec{\alpha}^{1-4}$, all projections are independent, and it is impossible to eliminate the $\mathbf{1 6}$ coming from this sector by altering the $k$-matrix. One can change the chirality of the $\mathbf{1 6}$ from one of these sectors, but again, this changes the net number of $N(\mathbf{1 6})-N(\overline{\mathbf{1 6}})$ by two. Clearly, if there is to be an odd number for $N(\mathbf{1 6})-N(\overline{\mathbf{1 6}})$, the number of sectors for which $n_{i}=7$ must be odd.

Unfortunately, it seems to be a generic property of basis vectors within this framework that massless sectors with $n_{i}=7$ always occur in sets of 4. We have not been able to construct examples for which this is not true. Thus, it does not seem possible to obtain three generation models based on $S O(10) \times S O(10)$ within our framework.

\section{$5 \quad S U(5) \times S U(5)$ Models}

We will attempt to get around this obstruction by breaking $S O(10)^{2} \rightarrow$ $S U(5)^{2}$. We note that if the vector $V_{P}$ is converted to a $1 / 4$ moded vector, breaking $S O(10) \rightarrow S U(5)$, the projection acting on a $\mathbf{1 6}$ will not leave a complete multiplet. If we replace $V_{P}$ with

$$
V_{7}=\left(0_{c} 0^{18} \mid 0^{12}+{ }_{c}^{16}\right)
$$

$\left(V_{P}=2 V_{7}\right.$, so all the above sectors are still here), the projection will be of the form

$$
\frac{1}{4} N_{5}=\sum_{i} \alpha_{i} k_{7 i} \quad(\bmod 1) .
$$

Depending on whether the right hand side equals $1 / 2$ or 0 , either a $\mathbf{1 0}$ or $1+\overline{\mathbf{5}}$ will survive. Of course, both a $\mathbf{1 0}$ and a $\overline{\mathbf{5}}$ are needed to make a complete family. Since string theory does not have nonabelian gauge anomalies, if a $\mathbf{1 0}$ arises in one sector, a $\overline{\mathbf{5}}$ (or $\overline{\mathbf{1 0}}$ ) must show up somewhere else ${ }^{5}$. In

\footnotetext{
${ }^{3}$ It is also possible that $(\mathbf{5 , 5})$ representations would appear as part of the anomaly cancellation. These would transform as $\mathbf{1 0}+\mathbf{1 5}$ 's under $S U(5)_{D}$, which is unacceptable phenomenologically. However, the appearance of these representations is easily prevented by an appropriate choice of the $k$-matrix.
} 
practice, it is easier to keep track of 10's; because two oscillator excitations are required to form a $\mathbf{1 0}$, these states generally only come from sectors with Ramond boundary conditions in the $S U(5)$ sector; i.e. the $\mathbf{1 6}$ sectors of the original $S O(10)$ model. Since only one excitation is needed for a $\overline{\mathbf{5}}$, these can come from a variety of new sectors which have $1 / 4$ boundary conditions in the $S U(5)$ sector and have $E_{L} \leq-1 / 4$.

Our strategy then is to construct an even generational $S O(10)^{2}$ model, break $S O(10)^{2} \rightarrow S U(5)^{2}$, and use the freedom of the $k$-matrix to control the number of 10's that appear in the original sectors of the $S O(10)$ model, allowing the string to fill in the remaining $\overline{\mathbf{5}}$ 's, such that a net total of three chiral families remain. Given that there are some 29 independent $k$-matrix elements, it does not seem unreasonable that one could do this.

Let us write down the $k$-matrix equations which determine whether a $\overline{5}$ or $\mathbf{1 0}$ survives in a given sector. We find:

$$
\begin{array}{ll}
\vec{\alpha}^{1}: & 0=k_{73} \\
\vec{\alpha}^{2}: & 0=k_{73}+k_{75}+k_{76} \\
\vec{\alpha}^{3}: & 0=k_{73}+k_{74} \\
\vec{\alpha}^{4}: & 0=k_{73}+k_{74}+k_{75}+k_{76}
\end{array}
$$

must be satisfied for a $\mathbf{1 0}$ to survive. The above equations are valid modulo 1. Recall that the above $k$-matrix elements must be 0 or $1 / 2$ (see Eq. 7). Unfortunately, we cannot find any set of $k_{i j}$ 's such that an odd number of the above equations are violated. This is because the above equations are not independent; adding the four of them gives zero mod 1. This is a direct consequence of the fact that $\sum_{1}^{4} \vec{\alpha}^{i}=0(\bmod 2)$, as can readily be seen from Eq. 23. The set of massless sectors with $n_{i}=7$ closes under addition. This fact prevents us from finding a projection in which an odd number of 10's survive. This property holds even if we modify $V_{7}$ to include additional $1 / 2$ boundary conditions for internal fermions. In all cases, we are stuck with an even number of generations.

To summarize, we have found that we are unable to break out of even numbers for $N(\mathbf{1 0})-N(\overline{\mathbf{1 0}})$, because various $k$-matrix equations degenerate, and this in turn follows from the fact that the set of sectors which produce massless 16's, both the nondegenerate and the degenerate ones, are separately closed under addition. This property seems to be common to all sets of basis vectors constructed within the framework of $S O(10)^{2}$ models with 
left-right pairing. We have not encountered a set of basis vectors based on Eq. 19 and preserving the $(\mathbf{1 0}, \mathbf{1 0})$ and the left-right pairing which does not have this property.

However, we have made some progress, in that now we know exactly what feature to look for in a set of basis vectors as we enlarge the scope of our search: we must look for an $S O(10)^{2}$ model for which the set of nondegenerate 16 generating sectors does not close on itself. We have chosen vectors which maintain a left-right pairing until now, because it provides a convenient way of finding solutions to Eqs. 3. However, this may be too restrictive. Notice that the triplet constraint, Eq. 11, imposes severe restrictions on right-hand side boundary conditions, and these restrictions are carried over to the left-hand side by the left-right pairing. We will now consider models which are made purely of complex fermions, with no leftright pairing imposed. We start with the same set as before, now written in purely complex notation:

$$
\begin{aligned}
& V_{2}=\left(\begin{array}{lllll}
1 & 100 & 010 & 010 \quad \mid & 1^{5} 0^{5} 000001000011
\end{array}\right) \\
& V_{3}=\left(\begin{array}{ll|lll}
1 & 010 & 100001 & 0^{5} & 1^{5} 000001001100
\end{array}\right)
\end{aligned}
$$

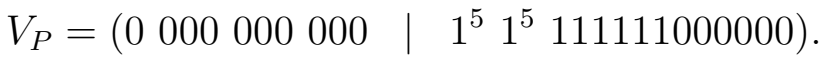

We must find three additional vectors which satisfy Eq. 3. Finding additional independent solutions of Eq. 3 becomes rather tedious as the number of basis vectors is increased, but can be done by computer. Here is a sample set of basis vectors which satisfies Eq. 3 and preserves the $(\mathbf{1 0}, \mathbf{1 0})$ Higgs:

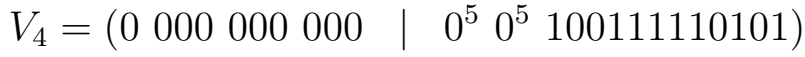

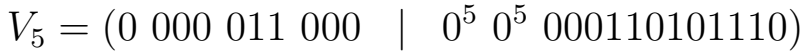

$$
\begin{aligned}
& V_{6}=\left(\begin{array}{lllll}
0 & 000 & 000000 \quad 0^{5} & 0^{5} & 111100001111
\end{array}\right) \text {. }
\end{aligned}
$$

This set gives the gauge group $S O(10)^{2} \times S U(2)^{2} \times U(1)^{10}$. The following sectors give massless, non-degenerate 16's:

$$
\begin{aligned}
& \vec{\alpha}^{1}=(0,0,1,0,0,0,0,0) \\
& \vec{\alpha}^{2}=(0,0,1,0,0,0,1,1) \\
& \vec{\alpha}^{3}=(0,0,1,0,0,1,1,1) \\
& \vec{\alpha}^{4}=(0,0,1,0,1,1,0,0) .
\end{aligned}
$$


The following sectors provide degenerate $\mathbf{1 6}$ 's:

$$
\begin{aligned}
\vec{\alpha}^{5} & =(0,0,0,1,0,0,0,0) \\
\vec{\alpha}^{6} & =(0,0,0,1,0,0,1,1),
\end{aligned}
$$

and $\mathbf{1 6}+\overline{\mathbf{1 6}}$ 's come from:

$$
\begin{aligned}
& \vec{\alpha}^{7}=(1,1,0,1,0,1,1,0) \\
& \vec{\alpha}^{8}=(1,1,0,1,1,1,0,1) .
\end{aligned}
$$

Once again, the number of non-degenerate $\mathbf{1 6}$ producing sectors is even, and it is impossible to obtain an odd number of $\mathbf{1 6}$ 's in an $S O(10)^{2}$ model. This is again seems to be true for all sets of basis vectors satisfying our constraints. However, there is an important difference between the above set of vectors and those of the previous example. One readily sees that

$$
\sum_{1}^{4} \vec{\alpha}^{i}=(0,0,0,0,1,0,0,0) \bmod 2 .
$$

The massless, nondegenerate sectors do not form a closed set by themselves, even though it is true that the larger set of massless sectors is closed; $\sum_{1}^{8} \vec{\alpha}^{i}=$ 0 mod 2. This removes the obstacle we had encountered before in finding projections which left an odd number of 10's, after breaking $S O(10)$ down to $S U(5)$.

Changing $V_{P}$ to $V_{7}$ as in Eq. (26),

$$
V_{7}=\left(0^{10} \mid+{ }^{10}++++++000000\right)
$$

the complete set of basis vectors is now

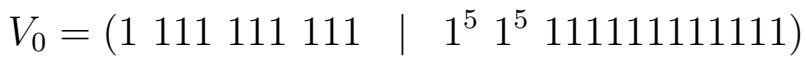

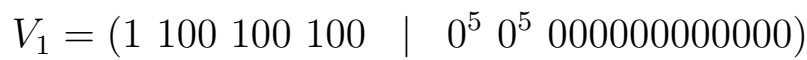

$$
\begin{aligned}
& V_{2}=\left(\begin{array}{llll|ll}
1 & 100 & 010 & 010 & 1^{5} & 0^{5} 000001000011
\end{array}\right) \\
& V_{3}=\left(\begin{array}{llll}
1 & 010100001 \quad \mid \quad 0^{5} 1^{5} 000001001100
\end{array}\right)
\end{aligned}
$$

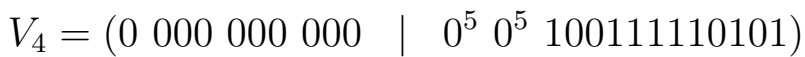

$$
\begin{aligned}
& V_{5}=\left(\begin{array}{ll|l}
0000011000 \quad \mid & 0^{5} 0^{5} 000110101110
\end{array}\right)
\end{aligned}
$$

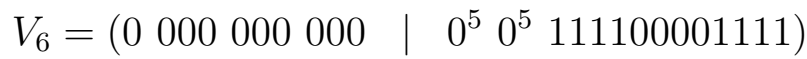

$$
\begin{aligned}
& V_{7}=\left(\begin{array}{ll|l}
0 & 000000000 \quad{ }^{5}{ }^{5}++++++000000
\end{array}\right) .
\end{aligned}
$$


Table 1. $S U(5) \times S U(5)$ Spectrum for Model 1.

\begin{tabular}{|l|l||l|l|}
\hline Sector & States & Sector & States \\
\hline \hline 00010000 & $2 \times(\mathbf{1 0}, 1)$ & 00100000 & $(1, \mathbf{1 0})$ \\
\hline 00100003 & $(1, \overline{\mathbf{5}})$ & 00100012 & $(\overline{\mathbf{1 0}}, 1)$ \\
\hline 00100013 & $(\mathbf{5}, 1)$ & 00100112 & $(\overline{\mathbf{5}}, 1)$ \\
\hline 00100113 & $(1, \overline{\mathbf{5}})$ & 00101100 & $(1, \mathbf{1 0})$ \\
\hline 00101103 & $(1, \overline{\mathbf{5}})$ & 01000001 & $(1, \overline{\mathbf{5}})+(\overline{\mathbf{5}}, 1)$ \\
\hline 01000003 & $(1, \mathbf{5})+(\mathbf{5}, 1)$ & 10110110 & $(\overline{\mathbf{5}}, 1)+(1, \mathbf{5})$ \\
\hline 10111000 & $(\overline{\mathbf{5}}, 1)+2 \times(1, \overline{\mathbf{5}})+(1, \mathbf{5})$ & 10111010 & $2 \times(\mathbf{5}, 1)+(1, \mathbf{5})+(\overline{\mathbf{5}}, 1)$ \\
\hline 10111110 & $(1, \mathbf{5})+(\overline{\mathbf{5}}, 1)$ & 10111111 & $(1, \overline{\mathbf{5}})$ \\
\hline 10111113 & $(\mathbf{5}, 1)$ & 11010111 & $(\overline{\mathbf{5}}, 1)$ \\
\hline 11010113 & $(\mathbf{5}, 1)$ & 11011101 & $(\overline{\mathbf{5}}, 1)$ \\
\hline 11011102 & $(\mathbf{1 0}, 1)+(\overline{\mathbf{1 0}}, 1)$ & 11011103 & $(\mathbf{5}, 1)$ \\
\hline 00000000 & $(\mathbf{5}, \overline{\mathbf{5}})+(\overline{\mathbf{5}}, \mathbf{5})$ & & \\
\hline
\end{tabular}

The $k$-matrix equations determining whether 10's or $\overline{5}$ 's survive in $\vec{\alpha}^{1}-\vec{\alpha}^{4}$ are:

$$
\begin{array}{ll}
\vec{\alpha}^{1}: & 0=k_{72} \\
\vec{\alpha}^{2}: & 0=k_{72}+k_{76} \\
\vec{\alpha}^{3}: & 0=k_{72}+k_{75}+k_{76} \\
\vec{\alpha}^{4}: & 0=k_{72}+k_{74}+k_{75} .
\end{array}
$$

It is now straightforward to find solutions which eliminate 10's from any sector we choose. For example, the choice $k_{74}=k_{75}=1 / 2$ (along with $k_{21}=k_{31}=1 / 2$ to preserve $N=1$ SUSY) eliminates 10's from $\vec{\alpha}^{3}$ while keeping them in the other three sectors. Actually, for this particular $k$ matrix, we find $(1, \mathbf{1 0})^{\text {'s }}$ in $\vec{\alpha}^{1}, \vec{\alpha}^{4}$ and a $(\overline{\mathbf{1 0}}, 1)$ in $\vec{\alpha}^{2}$, as well as $2 \times(\mathbf{1 0}, 1)$ from $\vec{\alpha}^{5}$ and $2 \times(1, \mathbf{1 0})$ from $\vec{\alpha}^{6}$, for a total $N(\mathbf{1 0})-N(\overline{\mathbf{1 0}})=5$. This is easily fixed by setting $k_{43}=1 / 2$, to eliminate $\vec{\alpha}^{6}$; the nonvanishing lower half diagonal $k$-matrix elements for this model are then

$$
k_{21}=k_{31}=k_{43}=k_{74}=k_{75}=\frac{1}{2} .
$$

The net total for $N(\mathbf{1 0})-N(\overline{\mathbf{1 0}})$ in this model is now three $(3 \times \mathbf{1 0}+\mathbf{1 0}+\overline{\mathbf{1 0}})$. Computing the complete massless spectrum for this model, we indeed find 
Table 2. $S U(5) \times S U(5)$ Spectrum for Model 2.

\begin{tabular}{|l|l||l|l|}
\hline Sector & States & Sector & States \\
\hline \hline 00010003 & $2 \times(\overline{\mathbf{5}}, 1)$ & 00010011 & $2 \times(1, \overline{\mathbf{5}})$ \\
\hline 00010012 & $2 \times(1, \overline{\mathbf{5}})$ & 00100000 & $(1, \mathbf{1 0})$ \\
\hline 00100003 & $(\mathbf{5}, 1)$ & 00100011 & $(1, \mathbf{5})$ \\
\hline 00100012 & $(\mathbf{1 0 ,}, 1)$ & 00100111 & $(\overline{\mathbf{5}}, 1)$ \\
\hline 00101100 & $(1, \mathbf{1 0})$ & 00101103 & $2 \times(\mathbf{5}, 1)$ \\
\hline 10110110 & $(1, \overline{\mathbf{5}})+(\mathbf{5}, 1)$ & 10110111 & $(\overline{\mathbf{5}}, 1)$ \\
\hline 10110113 & $(1, \mathbf{5})$ & 10111000 & $(\mathbf{5}, 1)+(\overline{\mathbf{5}}, 1)+(1, \mathbf{5})+(1, \overline{\mathbf{5}})$ \\
\hline 10111011 & $(1, \overline{\mathbf{5}})+(\overline{\mathbf{5}}, 1)$ & 10111013 & $(1, \mathbf{5})+(\mathbf{5}, 1)$ \\
\hline 10111110 & $(\overline{\mathbf{5}}, 1)+(1, \mathbf{5})$ & 11010110 & $(1, \mathbf{5})+(1, \overline{\mathbf{5}})$ \\
\hline 00000000 & $(\mathbf{5}, \overline{\mathbf{5}})+(\overline{\mathbf{5}}, \mathbf{5})$ & & \\
\hline
\end{tabular}

that the correct number of $\overline{\mathbf{5}}$ 's do appear in other sectors to cancel both $S U(5)$ anomalies. The total gauge group with this particular $k$-matrix is $S U(5) \times$ $S U(5) \times S U(4) \times S U(2) \times U(1)^{10}$ (the hidden sector gauge group is enhanced by twisted sector gauge bosons). In addition to the above states, there are an additional $12 \times(\mathbf{5}+\overline{\mathbf{5}})$ 's, 47 singlets charged under the $U(1)$ 's, and a variety of representations transforming under the hidden sector gauge group, from various sectors. The complete spectrum of $S U(5) \times S U(5)$ nonsinglet states is shown in Table 1.

With this set of basis vectors (31), we can now use the $k$-matrix to tailor the appearance of 10's more or less to our desires. For example, we can eliminate 10's coming from the degenerate sectors by choosing the $k$ 's to change them to 5's, and then alter the choice of $k$ 's to switch the $\overline{\mathbf{1 0}}$ in $\vec{\alpha}^{2}$ to a $\mathbf{1 0}$. Choosing

$$
k_{21}=k_{31}=k_{63}=k_{65}=k_{70}=k_{73}=k_{74}=k_{75}=1 / 2,
$$

we indeed find 10's from $\vec{\alpha}^{1,2,4}$ only, with the rest of the spectrum very similar to the previous example. The $S U(5) \times S U(5)$ nonsinglet spectrum for this model is shown in Table 2. To give a further example of the kind of freedom we have, suppose we wanted to have a model in which some sectors contained complete $1+\overline{\mathbf{5}}+\mathbf{1 0}$ multiplets. This can be accomplished by adding $1 / 2$ twists in $V_{7}$ such that complete multiplets survive from $\vec{\alpha}^{5,6}$, in the same manner as in Eq. 15. A careful examination of these sectors suggest the 
choice

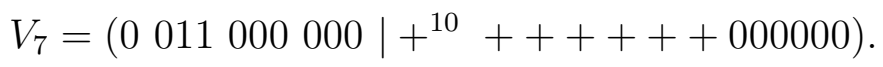

Along with a careful choice of $k$-matrix:

$$
k_{21}=k_{31}=k_{72}=k_{73}=k_{74}=k_{76}=1 / 2
$$

we find $1+\overline{\mathbf{5}}+\mathbf{1 0}$ 's in sectors $\vec{\alpha}^{5}, \vec{\alpha}^{6}$, and a $\mathbf{1 0}$ in sector $\vec{\alpha}^{4}$, with $\overline{\mathbf{5}}$ 's and 5's arising in various additional sectors, for a total of exactly three generations plus $14 \mathbf{5}+\overline{\mathbf{5}}$ pairs.

The set of basis vectors, Eq. 28, is not a unique set for which this method works. A computer search reveals a large number of solutions with the required property, that the non-degenerate massless sectors not form a closed set. It is not clear how many are genuinely distinct and how many are just relabellings and permutations of the others, but at least some are distinct because they contain numbers of degenerate massless sectors. For example, making the replacement

$$
V_{6}=\left(\begin{array}{lll|lll}
0 & 000000011 \mid 0^{5} 0^{5} 101101000011
\end{array}\right)
$$

in Eq. 28 leads to a set of basis vectors which has four non-degenerate $\mathbf{1 6}$ sectors and only two sectors with degenerate 16's, while the choice

$$
V_{6}=\left(\begin{array}{lllll}
0 & 011 & 011000 \mid 1^{5} 1^{5} 110000110011
\end{array}\right)
$$

leads to a model with six sectors providing degenerate $\mathbf{1 6}$ 's.

Much work needs to be done to see if these models are viable phenomenologically. These models all posess a large number of unwanted particles (14 $\mathbf{5}+\overline{\mathbf{5}}$ pairs and 50 or so singlets). Since these are in real $S U(5) \times S U(5)$ representations there is no obstacle in principle to their getting large masses after some moduli are given vacuum expectation values, although to actually see if this happens would require a detailed analysis of $\mathrm{F}$ and D-flat directions in these models. A preliminary look indicates that it would be very difficult to obtain a single large Yukawa coupling for the top quark in these models, and the question of dimension four operators baryon number violating operators is not clear. The most serious problem in supersymmetric grand unified theories, the doublet-triplet splitting for the Higgs bosons, has not been addressed. 


\section{Conclusions}

Within the free fermion constructions, we have seen that it is surprisingly difficult, if not impossible, to construct three generation models based on the $S O(10)$ group. This mirrors the results of other groups, pursuing different approaches. Whether there is any deep signifigance to this, or it is simply an artifact of the free fermion construction, is not clear.

We have, however, identified a way of constructing three generation models based on $S U(5)$. The number three does not arise naturally in these models, but arises only after a careful choice of projections. While the specific models constructed here do not appear to have any obvious phenomenological virtues, beyond the number of generations, they do demonstrate that there is at least one way around a barrier that has faced string GUT model

building. It will be interesting to see if these constructions can be turned into realistic grand unified theories.

\section{Acknowledgements}

It is a pleasure to thank M. Peskin for his encouragement and suggestions, L. Dixon for reading an early version of this manuscript, and J. Feng for participation in the early stages of this work. This work was supported in part by the Department of Energy under grant \#DE-AC03-76SF00515.

Note Added: After the completion of this work, 25] appeared, which addresses some related issues from the orbifold approach.

\section{References}

[1] S. Dimopoulos and H. Georgi, Nucl. Phys.B193(1981) 150;

S. Dimopoulos, S. Raby, and F. Wilczek, Phys. Rev. D24(1981) 1681;

N. Sakai, Zeit. Phys. C11 (1981) 153;

L. Ibanez and G.G. Ross, Phys. Lett. 105B(1981) 439;

M.B. Einhorn and D.R.T. Jones, Nucl. Phys. B196(1982) 475;

U. Amaldi et al., Phys. Rev. D36(1987) 1385;

U. Amaldi, W. de Boer, and H. Furstenau, Phys. Lett. 260B(1991) 447;

P. Langacker and M. Luo, Phys. Rev. D44(1991) 817.

[2] P. Ginsparg, Phys. Lett. 197B(1987) 139. 
[3] V.S. Kaplunovsky, Nucl. Phys. B307(1988) 145; Erratum, Nucl. Phys. B382(1992) 436.

[4] K. Dienes and A. Faraggi, Nucl. Phys. B457(1995) 409.

[5] J. Casas and A. Munoz, Phys. Lett B214(1988) 543;

L. Ibanez, Phys. Lett. B318(1993) 73;

K. Dienes, A. Faraggi, and J. March-Russel, hep-th/9510223.

[6] L. Dixon, V. Kaplunovsky and C. Vafa, Nucl. Phys. B294(1987) 43.

[7] H. Dreiner, J. Lopez, D. Nanopoulos and H. Reiss, Phys. Lett. 216B(1989) 283.

[8] D.C. Lewellen, Nucl. Phys. B337(1990) 61.

[9] S. Chaudhuri, S.-w. Chung, G. Hockney, and J. Lykken, Nucl. Phys. B456(1995) 89.

[10] A. Aldazabal, A. Font, L.E. Ibanez and A.M. Uranga, Nucl. Phys. B452(1995) 3.

[11] S-T Yau, in Symposium on Anomalies, Geometry and Topology, W. Bardeen, A. While eds., World Scientific, 1985.

[12] I. Antoniadis, J. Ellis, J. Hagelin, and D. V. Nanopoulos, Phys. Lett. 231B(1989) 65.

[13] I. Antoniadis, G. Leontaris, and J. Rizos, Phys. Lett. 245B(1990) 161.

[14] A. E. Faraggi, Phys. Lett. 278B(1992) 131; Phys. Lett. 339(1994) 223.

[15] P. Candelas, M. Lynker, and R. Schimmrigk, Nucl. Phys. B341(1990) 383.

[16] S. Kachru, Phys. Lett. 349B(1995) 76.

[17] A. Font, L.E. Ibanez and F. Quevedo, Nucl. Phys. B345(1990) 389.

[18] G. Cleaver, HEP-TH-9409096. 
[19] A.A. Maslikov, S.M. Sergeev, and G.G. Volkov, Phys. Lett. B328(1994) 319; Phys. Rev. D50 (1994) 7440; Int. J. Mod. Phys. A9 (1994) 5369; A.A. Maslikov, I. Naumov, and G.G. Volkov, hep-ph/9505318.

[20] R. Barbieri, G. Dvali and A. Strumia, Phys. Lett. 333B(1994) 79; Nucl. Phys. B435(1995) 102.

[21] H. Kawai, D.C. Lewellen and S.-H.H. Tye, Nucl. Phys. B288(1987) 1; H. Kawai, D.C. Lewellen, J.A. Schwartz and S.-H.H. Tye, Nucl. Phys. B299(1988) 431.

[22] I. Antoniadis, C.P. Bachas and C Kounnas, Nucl. Phys. B289(1987) 87.

[23] A. Faraggi and D. Nanopoulos, Phys. Rev. D48(1993) 3288;

I. Giannakis, D. Nanopoulos and K. Yuan, Phys. Rev. D52(1995) 1026.

[24] A. Faraggi, Phys. Lett. 326B(1994) 62.

[25] G. Aldazabal, A. Font, L.E. Ibanez and A.M. Uranga, FTUAM-95-27, HEP-TH-9508033. 